\title{
ICARO: A Computer Aided Diagnosis Tool for the Quantification of Intracoronary and Intravenous Echocardiography
}

\author{
D Sánchez-Perera ${ }^{1,2,3}$, D Moratal $^{1}$, DR García-Sánchez ${ }^{1}$, V Bodí-Peris $^{3}$, \\ JJ Rieta ${ }^{1}$, C Sánchez-Meléndez ${ }^{4}$ \\ ${ }^{1}$ Bioengineering, Electronics and Telemedicine Research Group, Universidad Politécnica de \\ Valencia, Spain \\ ${ }^{2}$ Universidad de Las Palmas de Gran Canaria, Spain \\ ${ }^{3}$ Cardiology Department, Hospital Clínico Universitario, Valencia, Spain \\ ${ }^{4}$ DIEEA, EUPCuenca, Universidad de Castilla-La Mancha, Spain
}

\begin{abstract}
The detection of coronary stenosis and myocardial viability with myocardial contrast echocardiography (MCE) has been based on the visual interpretation of the perfusion region by medical experts. To have a purely objective diagnostic result has been developed ICARO.

ICARO makes a complete analysis of an intracoronary or intravenous echocardiography (MCE-ic or MCE-iv) video sequence quantifying objectively the contrast uptake curves as well as the intensity levels on the myocardial wall among several other parameters.

Eighty cases have been analyzed using ÍCARO with more than $93 \%$ of concordance between our software and the visual diagnostic of three cardiologists.

ICARO gives all the necessary tools to make a complete analysis of echocardiography video sequence with intracoronary and intravenous injection obtaining objective and quantified parameters, and making the diagnostic independent of the cardiologist.
\end{abstract}

\section{Introduction}

The use of Myocardial Contrast Echocardiography (MCE) allows great results to diagnostic Acute myocardial infarction (AMI) and coronary stenosis. $[1,2,3]$. However, diagnostics have been based on the visual interpretation of the perfusion region by medical experts.

Nevertheless echocardiography images contain perfusion defects and image's artifacts. The detection of a perfusion defect means a correct diagnostic, however confuse both makes erroneous results. Only expert cardiologists can distinguish each one. This problem can be resolved with the use of quantification [4].

Using quantification, the influence of artifacts is reduced [5-7]. Also subjective diagnostics can be based on objective parameters placing the experience of the cardiologist in a second plane. At the same time results can be reused and saved in databases to be consulted in future times.

To help specialists and to give objective results, ICARO has been developed, a computer Aided Diagnosis Tool for the Quantification of Intracoronary and Intravenous Echocardiography.

\section{Methods}

The researches of Wei and others proved that there is a relation between coronary flow and blood coronary volume that can be measured by MCE with intravenous injection of the contrast agent [5]. When microbubbles have been destroyed by the ultrasound pulse, the variation of the video intensity ("Video Intensity", VI) was fitted to an exponential function as

$$
y=A \cdot\left(1-e^{-\beta t}\right)
$$

In an image $y$ is the Video Intensity (VI) on an instant of pulsation $t$. $A$ is a constant value called "plateau" that reflects the myocardial microcirculation state after a time $t$ and $\beta$ is the rate constant that determines the rate of rise of VI, $\beta$ is proportional to the mean velocity of the microbubbles. These authors proved that the myocardial flow is proportional to myocardial flow is proportional to the mean velocity of the microbubbles. These authors proved that the myocardial flow is proportional to the product of "plateau" and the slope $\beta$.

On the basis of quantification in MCE proposed by Wei and others, it was decided to develop a software that allowed obtaining semiautomatically the main parameters of the study of intravenous myocardial perfusion and intracoronary myocardial perfusion (MCE-IV and MCEIC) through segments and territories following the normalized model proposed by Cerqueira and others, that 
divides myocardial into 17 segments [8]. The software obtains exponential curves, to extract plateau level and slope $\beta$, to quantify myocardial blood flow for MCE-IV and Multitrigger studies. In case we are working with MCE-IC or triggered studies, the software will extract the mean value of myocardial wall for each segment in the maximum contrast level state, so microcirculation can be evaluated.

ICARO has been developed using $\mathrm{C}++$ with the programming environment Borland Builder v6.0. An easy-to-use and friendly interface has been developed to make the software as intuitive as possible.

The program is divided in three different modules:

The first module is a Video Editor. In this part of the program user can select which frame or frames wants to study. Also the user can create new videos composed of the frames in which he is interested, so next time it not will be necessary to select the frames to study. Options as previous views or real time examples are available. The Video Editor interface can be seen in figure 1.

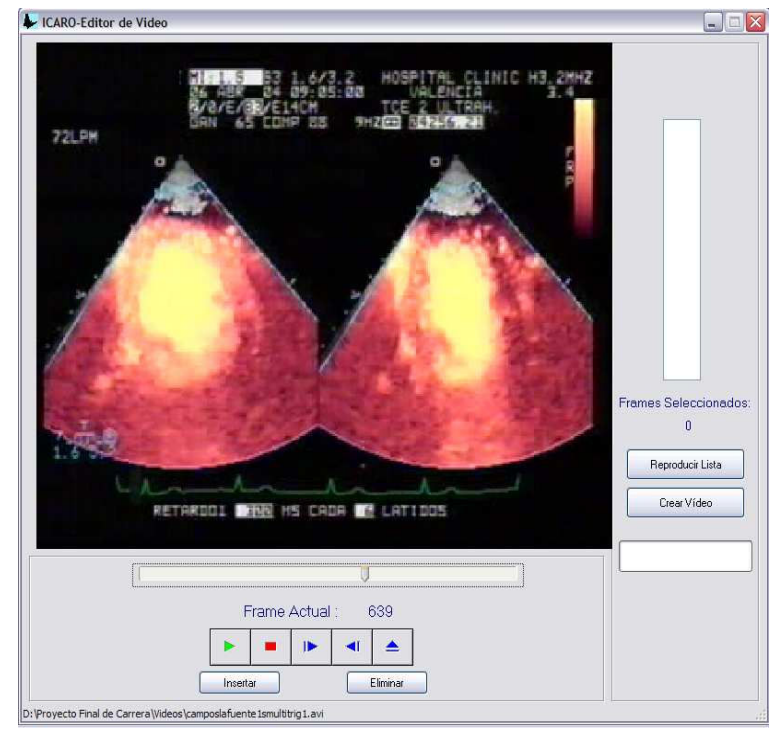

Figure 1 - ICARO's Video Editor

The second module is called Analisis Module. This is the brain of ICARO. Here MCE videos are quantified and parameters are extracted.

In this module, the user must select first what kind of video is being studied. Depending on what echocardiography view is selected, different available segments will be shown. Then the program makes a preprocessing of the frames to eliminate non homogeny attenuation normalizing segments respect the basal-state frame.

When preprocessing has been done, the user must select which segments and which regions of the segments wants to study. To do it, the user has to click in the interest zone of the image and video intensity will be saved.

Once all values have been capturated results are normalized to be shown so all segments intensity levels will be between [ $\left[\begin{array}{ll}0 & 1\end{array}\right]$.

In case the study is MCE-IC or trigger, normalization is made by a segment considered as normal. This segment could be designated automatically by the software (being the one with highest video intensity) or introduced by the user.

If the study is MCE-IV or multitrigger, user can select between two different normalizations. First normalization can be made by the segment that user considers as normal.

In other case, normalization responds at following expression:

$$
I_{n}=\frac{I_{s n}-I_{\min }}{I_{\max g}-I_{\min g}}
$$

$\left(I_{\text {maxg }}-I_{\text {ming }}\right)$ is the range of the segment which more increases its video intensity. $I_{\min }$ is the minimum value of the segment to normalize. $I_{s n}$ is the value to normalize. In this way, all segments video intensity are between [0 1 1] being the segment that has more perfusion the one which achieves 1 . Once the values are normalized they are fitted to the exponential curve and plateau level, slope $\beta$ and $\mathrm{A}^{*} \beta$ are extracted so myocardial microcirculation and myocardial perfusion level can be evaluated.

All this process is done with an intuitive and interactive interface.

As can be shown in the figure 3 the interface of the analysis module can be divided into six different regions.

A: Selection section. Here the user indicates ICARO where is the region to study for each segment. To make this work easier the tool allows zoom the image. The region that will be catch up is also configurable by the user.

B: Segments region. In this section the user selects which segments wants to study. The user only has to click on the segment and a mark will be shown to inform the user.

C: Studies section. In this dialog box the user selects the kind of study. Available segments will be shown and different options will be activated depending on the study selection.

D: Normalization section. The user will select which of the normalization wants to use to evaluate the results.

$\mathrm{E}$ : Undo section. If the user wants to make corrections he must select here the type of correction that wants to do. 


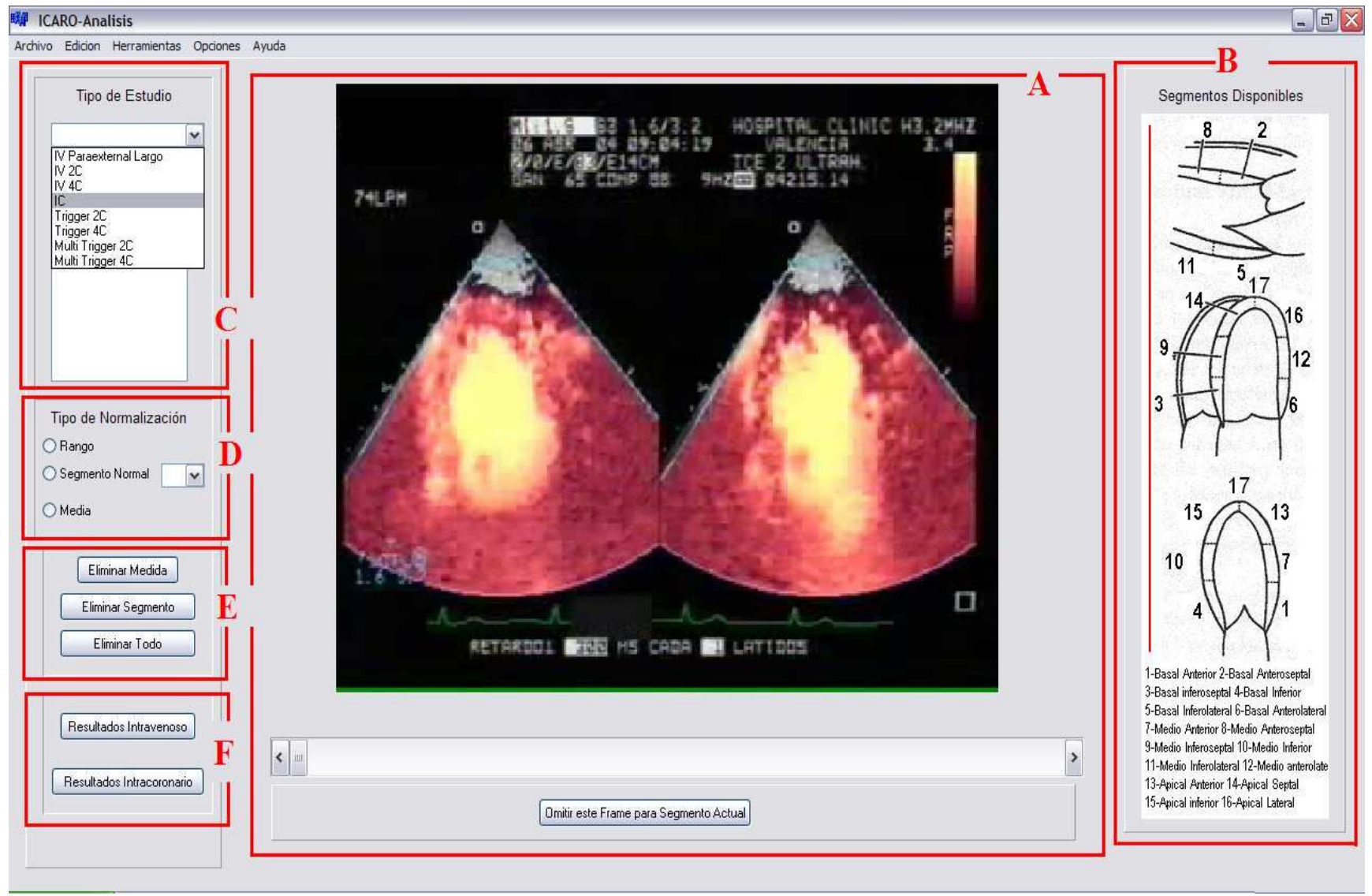

Figure 2 - ICARO's Analysis Module

F: Results section. Once the study is finished the user will indicate ICARO clicking here to pass to the third module to show the results in new windows.

The third module is the result module. In this module, results are shown as contrast uptake for each heart segment for MCE-IV or multitrigger studies. As can be seen in figure 3 the main parameters of these curves ("plateu" maximum level and curve slope among others) are also presented. Here the user can compare segments and evaluate their quantified parameters.

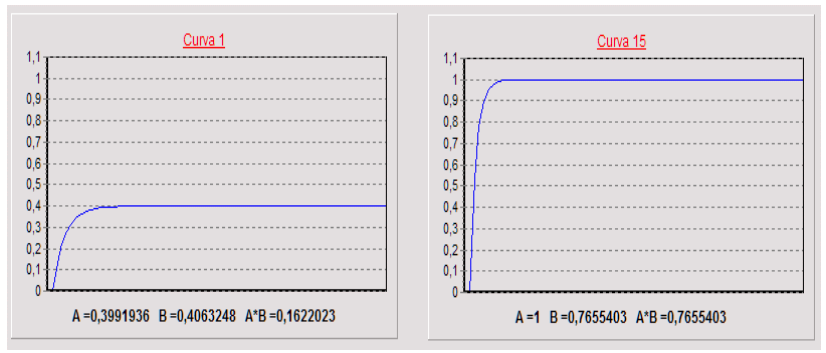

Figure 3 - Contrast uptake curves for segments 1 and 15 with the $\mathrm{A}, \mathrm{B}$ and $\mathrm{A} * \mathrm{~B}$ levels
For MCE-IC and triggered studies the results are represented as bar diagrams of contrast normalized intensity. As can be seen in figure 4 normal segment takes 1 and the other segments have proportional values.

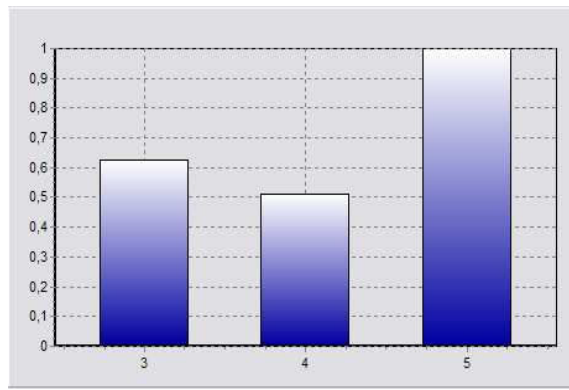

Figure 4-Bar diagrams of video intensity for segments 3, 4 and 5 


\section{Results}

ICARO has been tested by the Cardiology Departament of the Hospital Clinico Universitario of Valencia, Spain. Eighty cases have been analyzed using ÍCARO with more than $93 \%$ of concordance between our software and the visual diagnostic of three cardiologists. The $7 \%$ can be divided in $5 \%$ of false positives and $2 \%$ of false negatives. The time needed to make a complete study is about 3 minutes.

\section{Discussion and conclusions}

ICARO makes a complete analysis of an intracoronary or intravenous echocardiography (MCE-ic or MCE-iv) video sequence quantifying objectively the contrast uptake curves as well as the intensity levels on the myocardial wall among several other parameters.

The software gives objective results, without experts influence. ICARO is an easy-to-use software that, with a short time for a study, gives results with a great percentage of concordance.

ICARO is being used daily at Hospital Clínico Universitario of Valencia as a helpful tool in perfusion evaluation with great success.

\section{Acknowledgements}

This project has been partially subsidized by the action IIARC0/2004/249 of the Conselleria d'Empresa Universitat i Ciència de la Generalitat Valenciana.

\section{References}

[1] Ito H, Tomooka T, Sakai N, Yu H, Higashino Y, Fujii K, et al. Lack of myocardial perfusion inmediately alter succesful thrombolysis. A predictor of poor recovery of left ventricular function in anterior myocardial infarction. Circulation 1992;85:1699-705.

[2] Main ML, Magalski A et al. Usefulness of myocardial contrast echocardiography in predicting global left ventricular functional recovery alter anterior wall acute myocardial infarctaion, Am J Cardiol 2004;94:340-342

[3] Jiménez Borregueroa LJ, Ruiz-Salmerón R. "Valoración de la viabilidad miocárdica en pacientes con prerrevascularización". Revista Española de Cardiología 2003; 56(7):721-3

[4] Lindner JR, Sklenar J. Placing faith in numbers: quantification of perfusion with myocardial contrast echocardiography. J Am Coll Cardiol 2004;43:1814-6.

[5] Wei K, Jayaweera AR, Firoozan S, Linka A, Skyba DM, Kaul S. Quantification of myocardial Blood flow with ultrasound-induced destruction of microbubbles adminestered as a constant venous infusión. Circulation 1998;97:473-83.

[6] Swinburn JM, Lahiri A, Senior R. Intravenous myocardial contrast echocardiography predicts recovery of dysfunctional myocardium early after acute myocardial infarction. J Am Coll Cardiol 2001;38:19-25

[7] Shimoni S, Frangogiannis NG, Aggeli CJ, Shan K, Verani MS, Quinones MA, et al. Identification of hibernating myocardium with quantitative intravenous myocardial contrast echocardiography. Comparison with dobutamina echocardiography and Thallium-201 scintigraphy. Circulation 2003;107:538-44

[8] Cerqueira MD, Weissman NJ, Dilsizian V, Jacobs AK, Kaul S, Laskey WK, et al. Standardized myocardial segmentation and nomenclature for tomographic imaging of the heart. Circulation 2002;105:539-42. 